\title{
Internetabhängigkeit, Suizidalität und selbstverletzendes Verhalten - Ein systematisches Review
}

\section{Internet Addiction, Suicidality and Non-Suicidal Self-Harming Behavior - A Systematic Review}

\author{
Autoren \\ Toni Andreas Steinbüchel', Stephan Herpertz ${ }^{1}$, Ina Külpmann², Aram Kehyayan ${ }^{1}$, Jan Dieris-Hirche¹, \\ Bert Theodor te Wildt ${ }^{1}$
}

Institute

1 Klinik für Psychosomatische Medizin und Psychotherapie, LWL-Universitätsklinikum der Ruhr-Universität Bochum

2 Fakultät für Psychologie, Ruhr-Universität Bochum

\section{Schlüsselwörter}

Internetabhängigkeit, Suizidalität, Selbstverletzendes Verhalten, Prävalenz, Komorbidität

Key words

Internet addiction, suicidality, self injury, prevalence, comorbidity

eingereicht 22.05.2017

akzeptiert 12.09.2017

Bibliografie

DOI https://doi.org/10.1055/s-0043-120448

Online-Publikation: 23.11.2017

Psychother Psych Med 2018; 68: 451-461

(c) Georg Thieme Verlag KG Stuttgart · New York

ISSN 0937-2032

\section{Korrespondenzadresse \\ Toni Andreas Steinbüchel \\ Klinik für Psychosomatische Medizin und Psychotherapie, LWL-Universitätsklinikum der Ruhr-Universität Bochum Alexandrinenstraße 1-3 44791 Bochum \\ toni.steinbuechel@ruhr-uni-bochum.de}

Tab. 2 und 3 finden Sie unter https://doi.org/10. 1055/s-0043-120448

\section{ZUSAMMENFASSUNG}

Hintergrund Internetabhängigkeit (IA) geht mit einem hohen Maß an komorbiden psychischen Störungen, insbesondere Depressionen, Angststörungen, ADHS und Persönlichkeitsstörungen und einem erheblichen Leidensdruck einher. Im Hinblick auf das hieraus resultierende Gefahrenpotenzial untersucht die vorliegende Arbeit die aktuelle Forschungsliteratur bezüglich Suizidalität und nicht suizidalem selbstverletzendem Verhalten (NSSV) bei IA.

Methoden In einer systematischen Literaturrecherche wurden klinische und nicht-klinische Studien in 14 Datenbanken auf der Titel- Abstract-Ebene nach den häufigsten Schlagworten für IA, NSSV und Suizidalität durchsucht. Die nach Abzug der mehrfach vorhandenen Publikationen verbliebenen 2334 Artikel wurden nach definierten Ein- und Ausschlusskriterien gefiltert. Insgesamt konnten 15 Studien, die IA, NSSV und Suizidalität mittels validierter psychometrischer Instrumente erheben, in das Review eingeschlossen werden.

Ergebnisse Der Zusammenhang zwischen IA und Suizidalität wurde in 10 Studien untersucht, 4 Studien untersuchten den Zusammenhang von IA, Suizidalität und NSSV, eine Studie ausschließlich den Zusammenhang von IA und NSSV. Sämtliche Studien zeigten höhere Prävalenzen für NSSV beziehungsweise Suizidalität der Probanden mit einer IA im Vergleich zu Probanden ohne IA, wobei die Punkt-Prävalenzraten für Suizidalität mit 1,6-18,7\% erheblich variierten.

Diskussion Die Ergebnisse der eingeschlossenen Publikationen legen nahe, dass Internetabhängigkeit mit einer erhöhten Rate an nicht-suizidalem selbstverletzendem Verhalten und mit einer erhöhten Suizidalität einhergeht, wobei sich für Suizidgedanken ein deutlicherer Zusammenhang abbildet als für tatsächliche suizidale Handlungen. Um ein besseres Verständnis insbesondere kausaler Zusammenhänge zwischen IA, NSSV und Suizidalität zu entwickeln, bedarf es weiterer, möglichst longitudinaler Studien.

Fazit für die Praxis Vor dem Hintergrund der aktuellen Studienlage ist es ratsam, bei Internetabhängigen routinemäßig auch eine differenzierte Anamnese bezüglich NSSV sowie suizidalen Gedanken und Impulsen durchzuführen.

\section{ABSTRACT}

Background Internet addiction (IA) is associated with a high rate of co-morbid mental disorders, especially depression, anxiety disorders, ADHD and personality disorders and a considerable level of psychological strain. In terms of risk assessment, the present work investigates the current research literature on suicidal behavior and non-suicidal self-injurious behavior (NSSI). 
Methods We performed a systematic literature search in 14 databases on title and abstract level for the most common keywords for IA, NSSI and suicidality. After deduction of multiple items, 2334 articles remained. They were filtered per inclusion and exclusion criteria. We identified studies that examined the relationship between IA, NSSI and suicidality, which were assessed by validated psychometric instruments. This allowed a total of 15 studies to be included.

Results The relationship between IA and suicidality was examined in 10 studies, four studies examined the relationship of IA, suicidality, and NSSI, and one study exclusively focused on IA and NSSHB. All studies showed higher prevalence for NSSI and respectively suicidality of the subjects with an IA compared to subjects without IA, with point prevalence varying considerably between $1.6-18.7 \%$.

Discussion The results of the included publications suggest that Internet dependency is associated with an increased rate of non-suicidal self-harming behavior and increased suicidality, with suicidal ideation being more closely related to IA than suicidal actions. In order to develop a better understanding of causal relationships between IA, NSSI and suicidality, further longitudinal studies are required.

Conclusion Against the background of the presented studies NSSHB and suicidality need to be explicitly addressed within the assessment and treatment of IA patients.

\section{Einleitung}

Seit 2013 ist es zu einer erheblichen Zunahme der weltweiten Digitalisierung gekommen. Allein im Zeitraum von Dezember 2000 bis November 2015 hat die Internutzung weltweit um 832,5\% (Europa: 474,9\%) [1] zugenommen. Im Zuge dieser Entwicklung ist auch eine Zunahme des neuartigen Phänomens der Internetabhängigkeit zu verzeichnen [2]. Die negativen sozialen Auswirkungen der Sucht und ihre psychischen Begleiterkrankungen können zu einem erheblichen Leidensdruck führen, der sich auch in suizidalen Gedanken und Impulsen niederschlagen kann.

\section{Internetabhängigkeit}

Seit der ersten Publikation eines Fallberichtes im Jahre 1998 von K. Young zu einer Patientin, die einen Online-Chat-Dienst exzessiv nutzte [2], kam es zu einer deutlichen Zunahme der wissenschaftlichen Auseinandersetzung mit exzessivem und süchtigem Konsum von Online-Inhalten. Das neue Phänomen der exzessiven Internetnutzung entwickelte rasch eine medizinische Relevanz und führte zur Etablierung spezifischer therapeutischer Hilfsangebote, insbesondere Spezialambulanzen und -kliniken sowie Beratungsstellen [3]. Wenngleich die Internetabhängigkeit (IA) als eigenständige Diagnose bislang noch keinen Eingang in die Diagnosesysteme ICD 10 [4] und DSM 5 [5] gefunden hat, so ist zumindest ihre spezifische Variante als „online gaming disorder“ im Sinne einer Abhängigkeit von Online-Computerspielen als Forschungsdiagnose in den Anhang des DSM 5 aufgenommen worden (siehe Infobox). Insofern existiert auch noch keine Einheitlichkeit hinsichtlich der Nomenklatur. Zu den am häufigsten verwendeten Begriffen zählen neben der IA die Termini „compulsive Internet use“, „online addiction“, „problematic and pathological Internet use“, wobei sich global der Begriff „Internet addiction“ durchzusetzen scheint [3].

Als Kernmerkmale der Internetabhängigkeit werden, wenngleich bislang nicht einheitlich definiert, gedankliche Einengung [2, 5-8], Toleranzentwicklung [2, 5-7], sozialer Rückzug [2, 5, 6, 9], frustrane Abstinenzversuche mit Rückfällen [2, 5, 6, 9], Entzugserscheinungen (Irritabilität, Ängstlichkeit, Traurigkeit) [7, 9], Verlust am Interesse für frühere Hobbys oder Aktivitäten [5], Fortsetzung des exzessiven Konsums trotz des Wissens um die daraus resultierenden psychosozialen Probleme [5, 7], dysfunktionale Affektregulation [2, 6, 8], Belügen von Freunden, Familienmitgliedern oder Therapeuten zur Ver- heimlichung des tatsächlichen Konsums [2, 5] sowie der Verlust einer bedeutsamen Beziehung, eines Arbeits- oder Ausbildungsplatzes oder einer Karrierechance beschrieben [2, 5, 8, 9]. Ergänzend werden auch Ausschlusskriterien gefordert, insbesondere, dass die ausufernde Mediennutzung nicht als Ausdruck einer psychotischen Störung wie z. B. einer Manie [8] oder einer Zwangsstörung [7] zu verstehen ist. Die Dauer der täglichen Internetnutzungszeit ist für die Diagnostik relevant, stellt aber für sich genommen kein Kriterium für eine Internetabhängigkeit dar [10-13]. Die genannten Kernmerkmale sind meist für die Computerspielabhängigkeit benannt worden, sie sind jedoch auch auf die anderen Subtypen der Internetabhängigkeit übertragbar [3].

DSM-5 KRITERIEN FÜR DIE „INTERNET GAMING DISORDER“ IM APPENDIX DES DSM 5, DEUTSCHE VERSION [14]

Andauernder und wiederholter Gebrauch des Internets zum Zwecke des Online-Spielens, häufig gemeinsam mit anderen Spielern, der zu einer klinisch signifikanten Behinderung oder Einschränkung führt, gekennzeichnet durch 5 (oder mehr) der folgenden Symptome über eine Periode von 12 Monaten:

1) Andauernde Beschäftigung mit Internet- bzw. OnlineSpielen. (Der Betroffene denkt über frühere Online-Spiele nach oder beschäftigt sich gedanklich mit zukünftigen Spielen. Die Online-Spiele werden zur dominierenden Aktivität des alltäglichen Lebens.)

2) Entzugssymptome, wenn das Online-Spielen nicht zur Verfügung steht. (Diese Entzugssymptome werden typischerweise beschrieben als Gereiztheit, Ängstlichkeit oder Traurigkeit. Körperliche Symptome im Sinne eines Medikamentenentzugs werden nicht beschrieben.)

3) Toleranzentwicklung mit dem Bedürfnis, zunehmend Zeit für Online-Spiele aufzubringen.

4) Erfolglose Versuche, die Teilnahme an Online-Spielen zu beenden.

5) Verlust an Interesse an früheren Hobbys oder Aktivitäten als Folge des Online-Spielens.

6) Andauerndes exzessives Online-Spielen trotz des Wissens um die psychosozialen Probleme. 
7) Täuschen von Familienmitgliedern, Therapeuten oder anderen Personen in Bezug auf das wirkliche Ausmaß des Online-Spielens.

8) Gebrauch der Online-Spiele, um aus negativen Emotionen (wie z. B. Gefühle von Hilflosigkeit, Schuld oder Ängstlichkeit) herauszukommen oder um diese zu lindern.

9) Gefährdung oder Verlust von wichtigen Bekanntschaften, Beruf, Ausbildung oder Karriere-Möglichkeiten wegen des Online-Spielens.

Hinweis: Zu beachten ist dabei, dass sich die Internet Gaming Disorder von der Internet-Glückspielsucht unterscheidet, die im DSM 5 im Kapitel „Gambling Disorder (Pathologisches Spielen)“ aufgeführt wird.

Betrachtet man das gesamte Spektrum der Internetabhängigkeit ohne Unterteilung in ihre nach dem primären Konsum gegliederten Subgruppen (Computerspiele, Soziale Netzwerke, Cybersex, Online-Glückspiel und Online-Shopping), so findet sich eine große Varianz der Prävalenzraten. Bischoff et al. [15] ermittelten in einer 2012 durchgeführten repräsentativen deutschlandweiten $\mathrm{Er}$ hebung eine Punktprävalenz von $1 \%$ in der Altersgruppe der 14-64-Jährigen, wobei sich für die jüngere Teilstichprobe der 14 bis 24-Jährigen eine Punktprävalenz von 2,4\% ergab. In einer Übersichtsarbeit berichteten Carli et al. [16] unter Bezugnahme auf 5 Studien, die Jugendliche zwischen 12 und 18 Jahren im Zeitraum zwischen 1999 und 2008 untersuchten, über EU-weite Punktprävalenzen von 2-18,3\%. Für den asiatischen Raum wurde von diesen Autoren für die Zeiträume 2000 bis 2008 sogar eine noch gröBere Varianz der ermittelten Prävalenzraten von 0,9-37,9\% beschrieben. Demgegenüber lagen die Punktprävalenzangaben in den USA, auf der Basis von 2 inkludierten Erhebungen an Erwachsenen in den Jahren 2000 und 2004 zwischen 0,3-8,1\% [16].

Ungeachtet der verschiedenen Subgruppen und Prävalenzraten konnte bei den Betroffenen ein deutlicher Leidensdruck sowie relevante Einschränkungen und Beeinträchtigungen im Alltag durch die Unfähigkeit, den Internetkonsum zu steuern, sowie die hieraus entstehenden sozialen, beruflichen und gesundheitlichen Folgen (z. B. sozialer Rückzug und Isolation, Einschränkung der Leistungsfähigkeit mit Schul- oder Studienabbrüchen oder Arbeitsplatzverlusten, finanzielle Probleme) beobachtet werden [3]. Diese werden häufig durch vorbestehende oder im Verlauf entstehende Begleiterkrankungen verstärkt [17]. In Einzelstudien von Shapira et al. und Greenfield zeigte sich sogar bei $100 \%$ der Internetabhängigen mind. eine Begleiterkrankung $[18,19]$. Als charakteristische komorbide psychische Erkrankungen gelten Depressionen, Angststörungen, ADHS, substanzgebundene Abhängigkeit und Persönlichkeitsstörungen [16, 20-23]. Als auslösende und aufrechterhaltende Faktoren sind Persönlichkeitsmerkmale wie Impulsivität, geringer Selbstwert, Prokrastinationsverhalten, Einsamkeit sowie depressive und ängstlich-vermeidende Eigenschaften von Bedeutung [23]. Einige dieser Faktoren können gleichermaßen Prädiktoren für Suizidalität darstellen. Inwieweit bei Internetabhängigen störungsübergreifend Selbstverletzungen, suizidale Gedanken und
Verhaltensweisen eine Rolle spielen, ist bislang allerdings noch wenig untersucht.

\section{Suizidalität und selbstverletzendes Verhalten}

Nach O'Carroll et al. [24] ist Suizidalität als Summe aller Suizid-bezogenen Gedanken und Handlungen definiert und lässt sich in die Teilbereiche „suizidale Gedanken (ohne Handlungen)“, „nicht letal verlaufene Suizidversuche“ und „letal verlaufene Suizide“ unterteilen. Diese Einteilung wurde 2007 von Silverman et al. überarbeitet $[25,26]$. Die Autoren unterscheiden zwischen Suizid-bezogenen Gedanken, Suizid-bezogener Kommunikation (Suizidandrohungen, suizidalen Gesten im Sinne interaktioneller Handlungen mit gering-letalem Charakter) und Suizid-bezogenem Verhalten. Man geht davon aus, dass bei 87,3-90\% aller Personen, die suizidale Handlungen ausweisen, eine psychische Störung vorausgeht $[27,28]$.Eine aktuelle Studie von Joiner et al. [29] legt nahe, dass bei allen untersuchten Fällen, in denen die Probanden durch Suizid starben, zumindest eine subklinische Symptomatik einer psychischen Erkrankung vorlag. Insbesondere bei affektiven Störungen und Persönlichkeitsstörungen ist das Suizidrisiko erhöht. Bei den Abhängigkeitserkrankungen sind bei der Risikoabschätzung nicht nur substanzgebundene Abhängigkeitserkrankungen [30], sondern auch Verhaltenssüchte wie bspw. pathologisches Glücksspiel zu berücksichtigen [31]. Von den Suizidversuchen müssen die nicht-suizidalen selbstverletzenden Verhaltensweisen (NSSV) abgegrenzt werden, die zwar Handlungen mit bewusst intendierter und sozial unangemessener Selbstschädigung umfassen, jedoch nicht in der Absicht zu sterben oder den Tod billigend in Kauf zu nehmen [32]. NSSV treten meist bei Menschen mit hoher Impulsivität auf [33] und dienen oft der Emotionsregulation [34, 35].

Das Integrated motivational-volitional model of suicidal behavior beschreibt 3 Phasen in der Entwicklung suizidalen Verhaltens. In der prä-motivationalen Phase werden Hintergrundbedingungen und auslösenden Ereignisse zusammengefasst. In der sich anschließenden motivationalen Phase bildet sich aus Niederlagen und erlebten Demütigungen unter dem Einfluss subjektiv geringer Bewältigungsressourcen, Grübelschleifen, eingeprägten Vorurteilen ein Gefühl des Gefangenseins in der belastenden Lebenssituation heraus. Unter dem Einfluss motivationaler Moderatoren bilden sich suizidale Gedanken und Absichten heraus. Die Umsetzung suizidaler Absichten wird in der Willensphase von Faktoren wie der Impulsivität, Zugang zu geeigneten Mitteln, negativen Vorbildern, hoher Schmerztoleranz und geringer Ängstlichkeit beeinflusst [36]. Die interpersonal-psychological theory of suicidal behavior [37] postuliert, dass ein vermindertes Zugehörigkeitsgefühl, das Gefühl für andere eine Belastung zu sein, sowie eine Hoffnungslosigkeit bzgl. der Veränderlichkeit dieses Zustandes von der Fähigkeit des Individuums zum Suizid, die durch einen Habituationsprozess durch wiederholte Exposition körperlichen Schmerzen oder Furcht induzierenden Erfahrungen gegenüber, erworben werden kann, getrennt zu betrachten sind. Gerade das geringe Zugehörigkeitsgefühl im Hinblick auf realweltliche soziale Zusammenhänge, die sich auf erlebte Kränkungen und Verunsicherungen zurückführen lassen, können bei jungen Menschen zu lebensverneinenden Gedanken und/oder einem Rückzug in digitale Alternativwelten führen.

Gemäß den WHO World Mental Health Surveys [38] wird davon ausgegangen, dass Suizidversuche im Vergleich zu vollzogenen Su- 
iziden 20mal häufiger auftreten. Andere Studien gehen sogar von einer noch größeren Anzahl an Suizidversuchen aus, weswegen von einer hohen Dunkelziffer ausgegangen wird [39]. Die Weltgesundheitsorganisation (WHO) nennt in ihrem Aktionsplan „Preventing suicide: A global imperative “ [40] weltweite Inzidenzraten von 8,0 (Frauen) bzw. 15,0 (Männer) pro 100.000 Einwohner (für Europa: 4,9 [Frauen] bzw. 20,0 [Männer] pro 100.000 Einwohner), wobei es starke regionale und kulturelle Unterschiede gibt. In Europa ist der Suizid in der Gruppe der 10-24-Jährigen derzeit die zweithäufigste [40], bei 15-19-jährigen Frauen sogar die häufigste Todesursache [41]. Dies gilt es zu bedenken, wenn unter Jugendlichen und jungen Erwachsenen die Inzidenz der Internetabhängigkeit und die mit ihr assoziierten psychischen Störungen weiter steigt.

\section{Zusammenhänge zwischen Internetabhängigkeit, selbstverletzendem Verhalten und Suizidalität}

Die Zusammenhänge zwischen Internetnutzung und Suizidalität sind zunächst v. a. im Hinblick auf die Nutzung suizidbezogener Onlineforen untersucht worden [42]. Diese inhaltlich problematische Internetnutzung steht aber bislang in keinem empirisch nachgewiesenen Zusammenhang zu einer im quantitativen Sinne exzessiven pathologischen Internetnutzung.

Eine suchtartige Internetnutzung kann ein Ausdruck einer Flucht vor vermeintlich unlösbaren konkreten Schwierigkeiten im Lebensalltag sein, ohne dass suizidale Gedanken überhaupt ins Bewusstsein drängen. In diesem Sinne kann der pathologische Internetgebrauch nicht nur als neurotischer Konfliktlösungsversuch, sondern auch als Ausdruck einer dysfunktionalen Emotionsregulation dienen $[3,43,44]$. Die negativen körperlichen, sozialen und leistungsbezogenen Konsequenzen der IA können in Analogie zu den Folgen substanzbezogener Störungen insofern auch als selbstschädigendes Verhalten verstanden werden, womit sich eine Analogie zu den NSSV mit emotionsregulatorischer Funktion andeutet. Überhaupt gelten missbräuchliche und süchtige Verhaltensweisen und andere Störungen der Impulskontrolle als Risikofaktoren für suizidale Verhaltensweisen.

Impulsivität spielt bei der Pathogenese von IA, Suizidalität und NSSV auch unabhängig voneinander eine wichtige Rolle, da viele Handlungen aus einer Belastungssituation heraus ohne hinreichende Würdigung der langfristigen Konsequenzen erfolgen [33, 45, 46].

Der Kontrollverlust gegenüber dem Suchtmittel Internet wird von den Betroffenen oft als ein von außen kommendes und von ihnen selbst nicht beeinflussbares Geschehen wahrgenommen. Verunsicherung, Selbstwertprobleme und Hoffnungslosigkeit können die Folge sein, was im Sinne eines Circulus vitiosus den Internet-Konsum weiter verstärken kann. Im Zuge eines sozialen Rückzugs mit zunehmender Vereinsamung [47] entwickeln oder verstärken sich komorbide psychische Störungen, insbesondere Ängste und Depressionen, die ihrerseits mit Suizidgedanken einhergehen können [3]. Vor diesem Hintergrund ist insbesondere für die klinische Versorgung von Patienten mit IA die Frage relevant, ob IA selbst einen (protektiven oder eskalierenden) Einfluss auf suizidale Gedanken und Verhaltensweisen hat, oder ob die Suizidalität primär mit den charakteristischen komorbiden Störungen assoziiert ist. Im Hinblick auf diese Fragestellung ist die Metaanalyse von Franklin et al. [48] bedeutsam, die 5 entscheidende Prädiktoren für suizidale Gedanken ermittelt. 3 dieser Faktoren - Hoffnungs- losigkeit, Depression, Angststörungen - sind auch für die Internetabhängigkeit von Relevanz.

In 2 ersten Übersichtsarbeiten aus den Jahren 2011 [49] und 2013 [50] wurden die Ergebnisse von insgesamt 4 Studien zu IA, NSSV, Suizidalität und deren Zusammenhänge zusammengetragen. 2 dieser Studien [51, 52] verwendeten jedoch keine validierten Instrumente zur Erhebung von Suizidalität und NSSV, die beiden anderen Studien $[53,54]$ untersuchten lediglich mittels Suicidal Ideation Questionnaire (SIQ) die suizidalen Gedanken, jedoch keine NSSV oder suizidalen Impulse. Seitdem ist die Zahl der Studien im Web of Science, die sich mit den Zusammenhängen von IA, Suizidalität, NSSV, Suizidalität und psychischer Komorbidität beschäftigen, gestiegen (vgl. [3]), sodass es nun sinnvoll geworden ist, die aktuelle Datenlage erstmals im Rahmen eines umfassenden systematischen Reviews darzustellen. Dabei geht es v. a. um folgende Forschungsfragen: Tritt Suizidalität und NSSV bei Internetabhängigen tatsächlich gehäuft auf? Und welche Rolle spielen die charakteristischen Begleiterkrankungen von Internetabhängigkeit für die Suizidalität? - Die Ergebnisse sollen schließlich dazu dienen, die Berücksichtigung von Suizidalität in Diagnostik und Therapie zu verbessern.

\section{Material und Methoden}

Eine elektronische systematische Literaturrecherche wurde im Juni 2016 in den Datenbanken Academic Search Premier, Annual Reviews, Cochrane Library of Systemtic Reviews, DARE, EPub Ahead of Print, LILACS, Medline, NIHR Centre for Reviews and Dissemination, OVID Healthstar, OVID Medline EPub Ahead of Print, PsychArticles, Psyclnfo, PubPsych, ScienceDirect und Web of Science durchgeführt. Unter Suchbegriffen aus den Bereichen Internetabhängigkeit und Suizidalität ${ }^{1}$ in Keyword und Abstracts wurde nach Artikeln in deutscher oder englischer Sprache gesucht, die seit 1992 (Einführung des öffentlichen Internetzugangs) in Form von wissenschaftlichen Artikeln oder Buchbeiträgen publiziert wurden.

Nach Korrektur mehrfach vorhandener Artikel (2529) verblieben von den initial 4863 Artikeln noch 2334 Artikel. Es wurden ausnahmslos Studien eingeschlossen, die den Zusammenhang zwischen IA, NSSV und Suizidalität an klinischen oder nicht-klinischen Stichproben mittels validierter Instrumente untersuchten. Ausschlusskriterien waren: Kommentare (18), Studien zu IA-bezogenen Tests (65) und Therapieverfahren (44), zu neurophysiologischen Veränderungen (82), zu web-basierten diagnostischen oder therapeutischen Verfahren (257), Indizes sowie Poster- und Vortragszusammenfassungen (75) sowie Artikel, deren Volltext nicht in englischer oder deutscher Sprache vorlagen (180) oder ohne thematischen Bezug zu den Fragestellungen dieser Untersuchung (1581) waren. Letztendlich wurden 32 Artikel einer Volltextanalyse unterzogen. Nach Korrektur der Studien, die nicht mittels validierter psychometrischer Instrumente vorgegangen sind, verblie-

\footnotetext{
1 “Internet addiction" OR “Internet dependency" OR “Internet misuse" OR “Internet use" OR "online addiction" OR "problematic gaming" OR "gaming disorder" OR "gaming addiction" OR "problematic gaming" OR "excessive online gaming" OR "cybersex* addiction" OR "* phone * addiction" OR "social media addiction") AND (suicid* OR self* harm* OR self* injur* OR self* destruct* OR auto * aggre * OR depress * OR agression OR hostility* OR impulsiv* ) NOT (web * based OR internet * based)
} 
ben 15 Artikel. Um die Vergleichbarkeit der in den Studien verwendeten Instrumente zu erhöhen, werden diese in den Ergebnistabellen kurz beschrieben. In $\triangleright$ Abb. $\mathbf{1}$ ist die Systematik der Literaturrecherche dargestellt.

Die Studien wurden unterschieden nach IA-Subgruppen (Computerspiel-, soziale Netzwerkseiten-, Cybersex-, Onlineshopping-, Onlineglücksspiel- oder generalisierte Internetabhängigkeit), nach Studiendesign (Längs- oder Querschnittsuntersuchung) und nach Art der Datenerhebung (strukturiertes Interview oder psychometrische Instrumente).

\section{Ergebnisse}

In die Analyse gehen 15 Originalarbeiten [54-61] ein. Mit Ausnahme der Studie von Fu et al. [53] mit 2 Erhebungszeitpunkten stellen alle Studien Querschnittsuntersuchungen dar. Eine Zusammenfassung der Studienmerkmale findet sich in > Tab. 1.
Zehn Studien [53-57, 59-63] untersuchten den Zusammenhang von IA und Suizidalität, wobei sich 3 Studien [53-55] auf suizidale Gedanken beschränkten, während in der Studie von Kurt [57] nicht zwischen suizidalen Gedanken und Verhaltensweisen differenziert wurde. Die übrigen 6 Studien untersuchten sowohl suizidale Gedanken wie auch suizidales Verhalten [56, 59-63]. Die Zusammenhänge zwischen IA, NSSV und Suizidalität wurden in 4 Studien untersucht [64-67]. Die Studie von Oktan [58] untersuchte ausschließlich den Zusammenhang von IA und NSSV. Komorbide Störungen wurden in 11 Studien [53, 54, 56, 59-67] berücksichtigt.

Als Instrumente zur Erhebung der Internetabhängigkeit wurden der Internet Addiction Test (IAT) [55, 56, 62], die Internet Addiction Scale (IAS) $[54,57]$, der Young's Diagnostic Questionnaire (YDQ) $[53,64,65,67]$, die Chen Internet Addiction Scale (CIAS) in ihrer ursprünglichen [63] sowie in ihrer revidierten Version [61], die Problematic Internet Use Scale (PIUS) [58], der Problematic Cellular

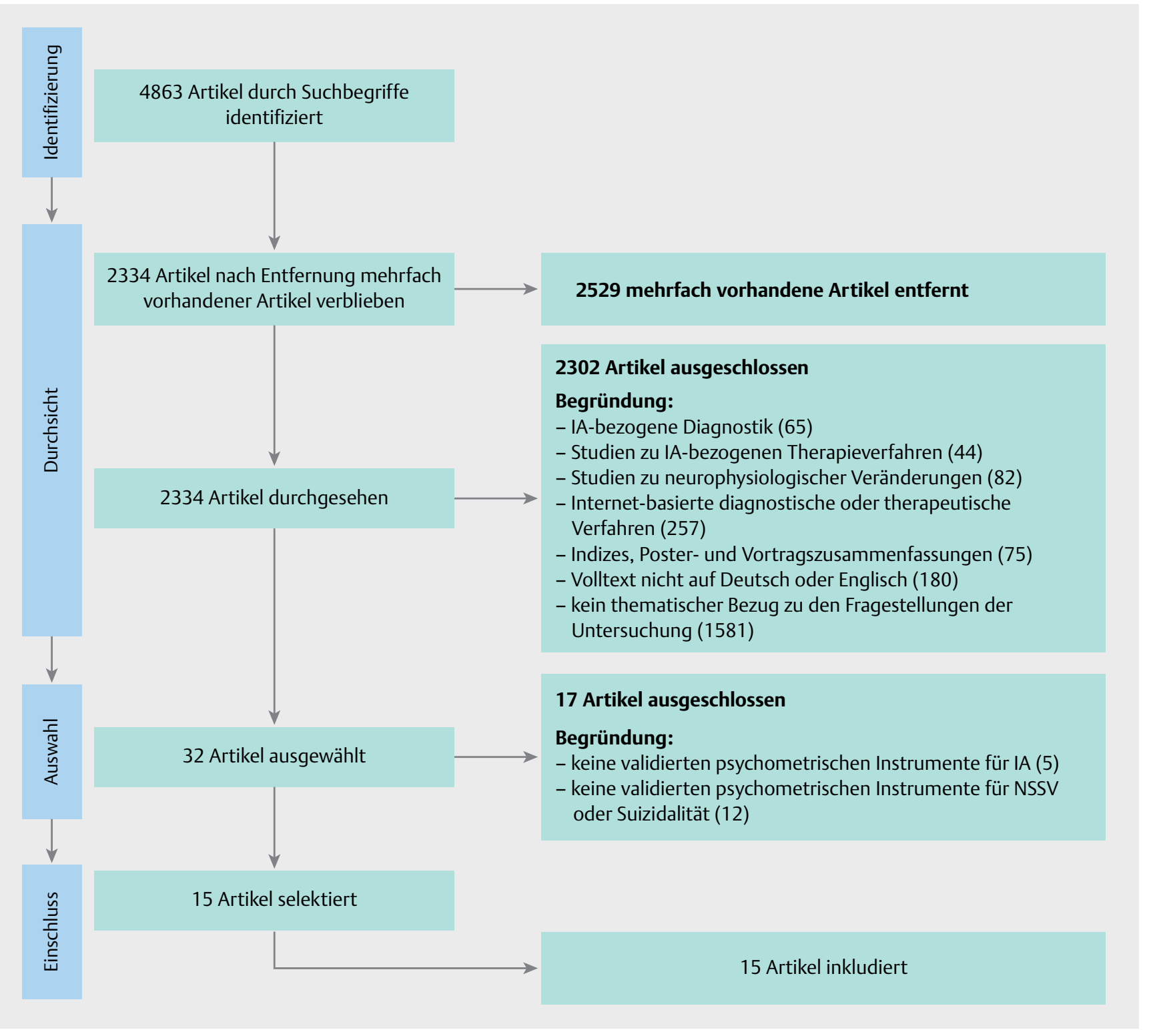

Abb. 1 Ablaufschema der Artikelselektion. 

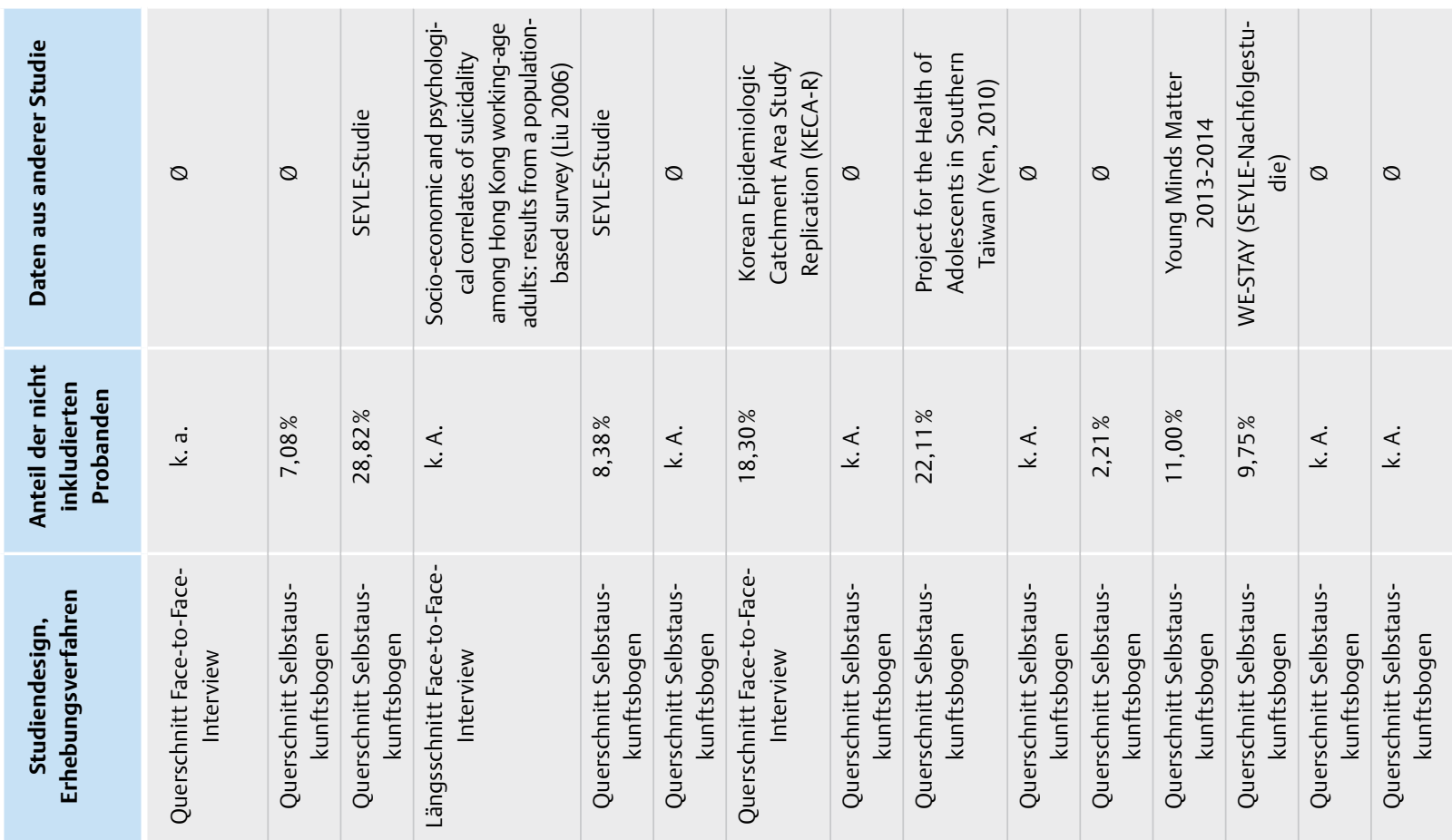

\begin{tabular}{|c|c|c|c|c|c|c|c|c|c|c|c|c|c|c|}
\hline 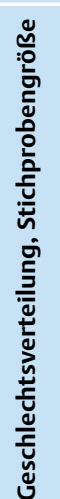 & 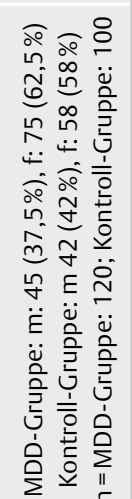 & 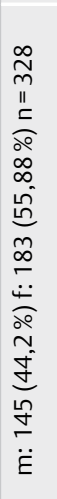 & 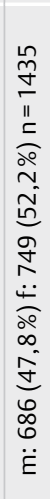 & 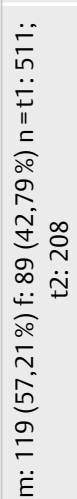 & 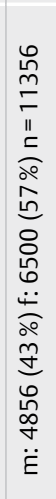 & 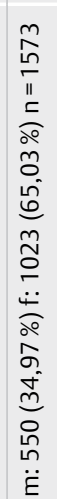 & 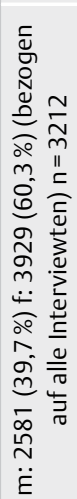 & 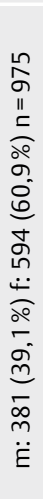 & 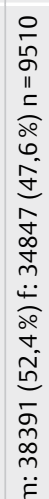 & 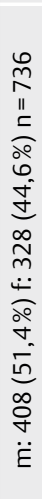 & 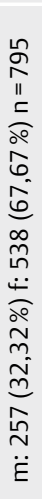 & 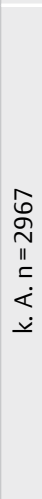 & 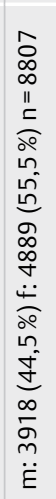 & 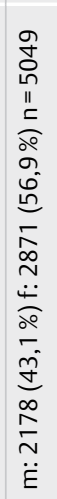 \\
\hline
\end{tabular}

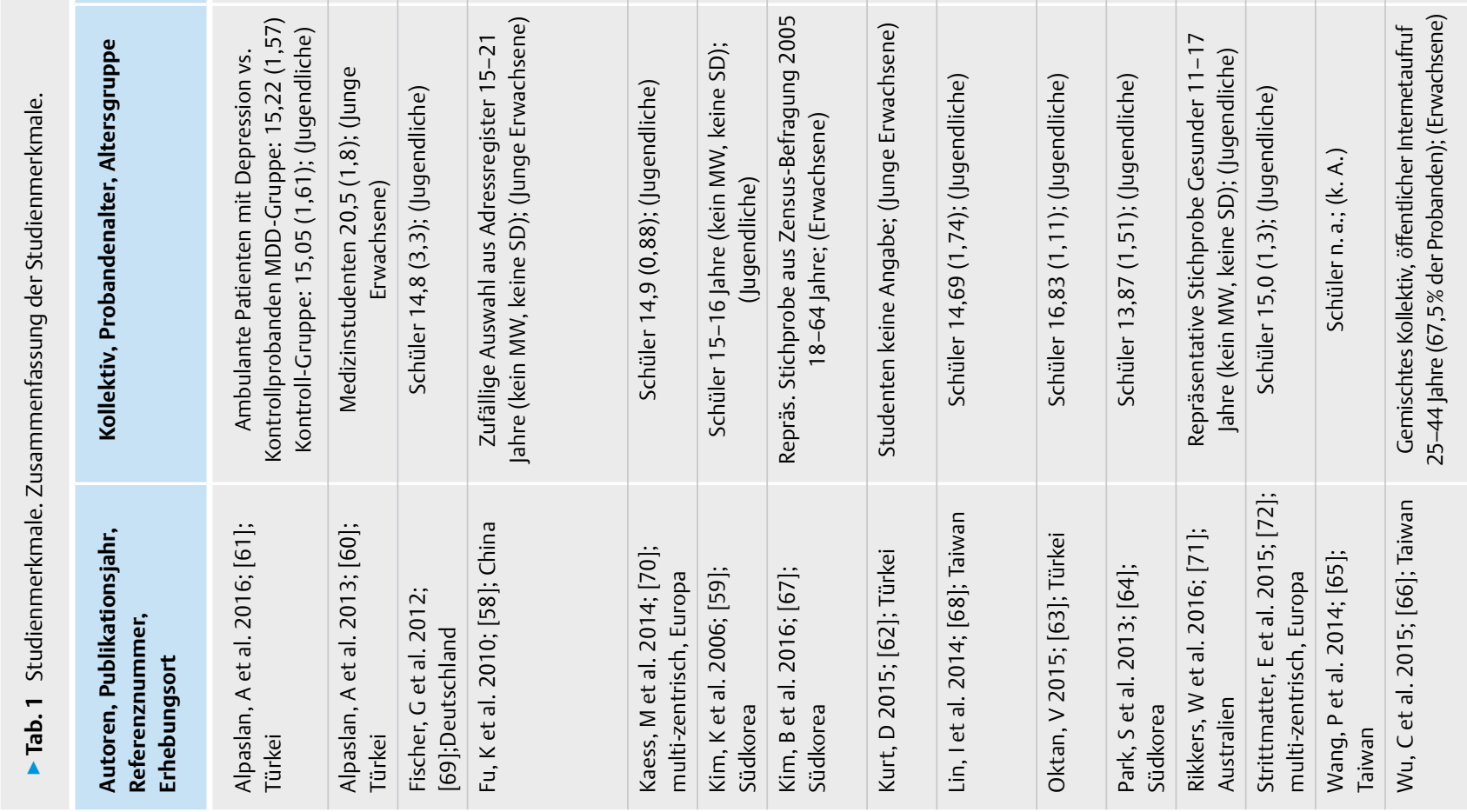


Phone Use Questionnaire (PCPU-Q) [60], die Korean Internet Addiction Proneness Scale for Youth-Short Form (KS Scale) [59] sowie angepasste Subskalen aus dem EU Kids Online Survey [66] verwendet. Hierbei ist zu berücksichtigen, dass es sich bei Young's Diagnostic Questionnaire (YDQ) und dem Internet Addiction Test (IAT) nicht um validierte Selbstauskunftsbögen, sondern um klinische Tests handelt, die für die jeweiligen Studien in Selbstauskunftsbögen umgewandelt wurden. Da diese Tests jedoch als gut validiert gelten, wurden sie für diese Untersuchung mit den anderen Instrumenten gleichgesetzt.

Die Untersuchung des NSSV stützten sich auf das Deliberate Self-Harm Inventory (DSHI) $[64,65,67]$ das Youth Risk Behavior Surveillance System (YRBSS) [66] oder den Adolescent Risk Taking Questionnaire (ARQ) [58]. Suizidale Gedanken und Verhaltensweisen wurden mittels der Paykel Suicide Scale (PSS) $[64,65,67]$, dem Suicidal Ideation Questionnaire (SIQ) $[53,59]$ beziehungsweise dem Suicidal Ideation Questionnaire - Junior (SIQ-JR) [54], der University Form of Risk Behaviors Scale (UFRBS) [57], der Five-Item Brief Symptom Rating Scale (BSRS-5) [61], Subskalen der Kiddie Schedule for Affective Disorders and Schizophrenia (Kiddie-SADSE) $[60,63]$ und der Suicide Prevention Multisite Intervention Study on Suicidal Behavior (SUPRE-MISS) [62] untersucht. Eine Übersicht über die verwendeten psychometrischen Instrumente findet sich in $>$ Tab. 2 im Internet.

Ausschließlich die Studie von Alpaslan et al. [56] untersuchte eine klinische Stichprobe mit Kontrollgruppe, alle anderen Studien basierten auf nicht-klinischen Stichproben. In 4 Studien [53, 62, 66] erfolgte die Rekrutierung über öffentliche Aufrufe oder Register, in 2 Studien $[55,57]$ wurden die Probanden direkt an Bildungseinrichtungen rekrutiert. Die Studie von Strittmatter et al. [67] untersuchte den Subtyp Computerspielabhängigkeit, die Studie von Wang et al. [60] den problematischen Smartphone-Gebrauch, wobei die Art der Anwendungen (Spiele, soziale Netzwerkseiten, Cybersex, etc.) nicht weiter spezifiziert wurde. Alle übrigen Studien leisteten keine differenzielle Auswertung bezüglich der IA-Subtypen. An statistischen Verfahren kamen v. a. Berechnungen von Quotenverhältnissen [57, 58, 63, 64, 67], Mittelwertvergleiche im Sinne von TTests [53-56, 59,60] und die Ermittlung von Prävalenzen $[61,62,65]$ zur Anwendung, wobei auf die Unterschiede zwischen Lebenszeit- und Punktprävalenzangaben zu achten ist.

Für die Punktprävalenz von IA ergab sich eine hohe Varianz zwischen 1,6-18,7\%, welche v. a. die unterschiedlichen Ergebnisse der asiatischen Studien, die mehrheitlich Jugendliche ab dem 15. Lebensjahr untersuchten, abbildet ( $\triangleright$ Tab. 3 im Internet). Die Varianz bei den 3 Studien im kontinentaleuropäischen Raum, die Jugendliche ab dem 11. Lebensjahr untersuchten, lag mit 3,1-4,8\% deutlich niedriger. Eine geschlechtsspezifische Auswertung hinsichtlich der Punktprävalenz für Internetabhängigkeit wurde lediglich in den Studien von Fischer et al. [64] (männliche Probanden: 4,8\%; weibliche Probanden: 4,8\%) und Park et al. [59] (männliche Probanden: $12,1 \%$; weibliche Probanden: $8,2 \%$ ) durchgeführt.

\section{Nichtsuizidales selbstverletzendes Verhalten und Internetabhängigkeit}

Zusammenhänge zwischen IA und NSSV wurden in 5 Studien an ausschließlich jugendlichen Probanden (11-18 jahre) untersucht $[64,65,67]$. Insgesamt kommen die Studien zu folgenden Ergeb- nissen. Es wurden gegenüber Vergleichsprobanden signifikant höhere Prävalenzraten des NSSV für IA allgemein (22,2/4,5\%) [65], für Computerspiel-IA (38,5/18,05\%) und für Nicht- ComputerspielIA $(49,1 / 18,05)$ [67] ermittelt. NSSV zeigte sich in einer Studie [58] als ein Prädiktor für Internetabhängigkeit. Zudem korrelierten die Maße für Internetabhängigkeit und NSSV signifikant positiv miteinander [58]. Entsprechende Zusammenhänge zeigten sich in den Quotenverhältnissen nicht nur für ein süchtiges Nutzungsverhalten, sondern auch für eine missbräuchliche Nutzung im Sinne eines Risikoverhaltens [58].

\section{Suizidale Gedanken und Internetabhängigkeit}

In 12 der 15 eingeschlossenen Studien wurden Zusammenhänge zwischen IA und suizidalen Gedanken untersucht, auf deren wesentliche Aspekte im Folgenden eingegangen wird ( $\triangleright$ Tab. 3 im Internet). Mit Ausnahme einer Studie ergaben sich in allen Studien signifikante Zusammenhänge zwischen suizidalen Gedanken und IA.

Bei Alpaslan et al. [56] bildete sich diese Beziehung unter der Kontrolle von Geschlecht, Alter und Depressivität nicht ab. Die Punktprävalenz von suizidalen Gedanken bei IA variierte bei den Studien zwischen 21,6\% [62] und 47,0\% [61]. In 2 europäischen Studien $[64,65]$ zeigten sich in den Gruppen mit IA signifikant höhere Prävalenzen $(p<0,001)$ für suizidale Gedanken $(27,5$ bzw. $42,3 \%$ ) verglichen mit den jeweiligen Gruppen ohne IA (10,9 bzw. 12,7\%). Lediglich eine Studie [62] untersuchte die Lebenszeitprävalenz für suizidale Gedanken, die in der Gruppe der Probanden mit IA (21,6\%) im Vergleich zu den Probanden ohne IA (14,0\%) ebenfalls signifikant erhöht war. In einer anderen Studie [59] konnte IA als signifikanter Prädiktor für suizidale Gedanken nachgewiesen werden $(p<0,05)$.

\section{Suizidales Verhalten und Internetabhängigkeit}

Ein Zusammenhang zwischen IA und konkretem suizidalem Verhalten wurde in 11 der 15 eingeschlossenen Studien erhoben. Mit Ausnahme von 3 Studien, bei denen die vergleichsweise hohen Werte für Suizidalität kein Signifikanzniveau erreichten [56], ließ sich jeweils ein signifikanter Zusammenhang zwischen IA und suizidalem Verhalten nachweisen. Die 3 an jugendlichen Probanden durchgeführten Studien $[64,65,67]$ konnten bei einer Varianz der Punktprävalenzraten für IA zwischen 3,1 \% [67] und 4,8 \% [64] jeweils signifikant höhere Prävalenzen von Suizidversuchen bei den Betroffenen identifizieren. In der Studie von Strittmatter et al. [67] zeigte sich dieser Unterschied nicht nur für Internetabhängige im Allgemeinen, sondern auch für die Online-Computerspielabhängigen im Besonderen.

Psychische Komorbiditäten von IA wurden in 13 der 15 eingeschlossenen Studien untersucht, wobei die Effekte der Komorbidität auf Suizidalität kaum differentiell betrachtet wurden. Die für IA charakteristischen Begleiterkrankungen Depressionen [53, 54, 56, 59-65, 67] ADHS [62, 65-67], Angststörungen [62] und substanzgebundene Abhängigkeitserkrankungen [62] bildeten sich auch in den hier zusammengestellten Untersuchungen ab. Lediglich für den Konsum illegaler Drogen konnte in der Studie von Park et al. [59] ein direkt verstärkender signifikanter Effekt auf das Suizidrisiko nachgewiesen werden. 


\section{Diskussion}

Die aktuelle Studienlage deutet darauf hin, dass Internetabhängigkeit mit einer erhöhten Rate an nicht-suizidalem selbstverletzenden Verhalten und mit einer erhöhten Suizidalität einhergeht, wobei sich für suizidale Gedanken ein deutlicherer Zusammenhang abbildet als für tatsächliche suizidale Handlungen. Wenn nun im Folgenden die Ergebnisse für die 3 Dimensionen Selbstgefährdung diskutiert werden, geschieht dies auch vor dem Hintergrund der Beeinflussung durch die charakteristischen Begleiterkrankungen von Internetabhängigkeit.

\section{Selbstverletzendes Verhalten bei Internetabhängigkeit}

In allen 5 Studien, die den Zusammenhang von NSSV und IA untersuchten, zeigten sich signifikant höhere Werte für Selbstverletzungen. Dieser Zusammenhang zeichnet sich stärker für die Abhängigkeit von Sozialen Medien als für die von Online-Computerspielen ab [3]. In epidemiologischen Studien deutet sich an, dass zunehmend mehr Mädchen und junge Frauen von dieser spezifischen IA betroffen sind, wenngleich sich dies bislang kaum im Klientel der Spezialambulanzen abbildet. Dies könnte sich eventuell damit erklären lassen, dass Internetabhängigkeit häufig übersehen werden kann, wenn sie im Rahmen von Erkrankungen mit hoher Impulsivität auftritt, welche sich sowohl in süchtigem als auch anderweitig selbstverletzendem Verhalten zeigen kann. Wenngleich es bisher kaum Untersuchungen zu Persönlichkeitsstörungen bei IA gibt, so zeigen jüngere Arbeiten v. a. bei weiblichen Betroffenen einen Zusammenhang zwischen Borderline-Störungen und abhängigem Internetnutzungsverhalten [68, 69]. Sollte sich im Zuge weiterer Studien der Verdacht auf einen Zusammenhang zwischen IA und NSSV erhärten, ließe sich störungsübergreifend ein möglicher Erklärungsansatz im Persönlichkeitsmerkmal Impulsivität finden, das sowohl bei Internetabhängigen als auch bei Menschen mit selbstverletzendem Verhalten wiederholt nachgewiesen werden konnte $[16,70]$. In jedem Fall erscheint bereits jetzt eine Mediennutzungsanamnese bei Menschen mit emotional-instabilen Persönlichkeitsstörungen als sinnvoll, um auch in der Therapie etwaigen Verschiebungen impulsiven Verhaltens Rechnung tragen zu können.

\section{Suizidale Gedanken bei Internetabhängigkeit}

In fast allen Untersuchungen, in denen explizit suizidale Gedanken erhoben wurden (10 von 11), ließen sich signifikante Zusammenhänge zwischen Internetabhängigkeit und Suizidgedanken nachweisen. Die Ergebnisse sprechen dafür, dass Menschen mit IA häufiger unter suizidalen Gedanken leiden. Es ergaben sich bei den Internetabhängigen für Suizidalität entweder signifikant erhöhte Prävalenzen oder ein höheres Maß an Suizidalität. Diese Ergebnisse müssen im Kontext der charakteristischen Begleiterkrankungen von IA interpretiert werden. Alle elf Studien zeigen höhere Prävalenzraten für depressive Störungen beziehungsweise eine ausgeprägtere depressive Symptomatik in den IA-Gruppen. Wie in anderen Übersichtsarbeiten, die sich ausschließlich mit der Komorbidität von IA befassen (z. B. Carli et al. [16], Ko et al. [21] oder Lee et al. [22]), zeigten sich neben ADHS, Angststörungen und substanzgebundener Abhängigkeit v. a. depressive Symptome und Episoden als charakteristische Begleitphänomene. In den WHO World
Mental Health Surveys [71] litten Erwachsene mit Suizidalität durchschnittlich am häufigsten unter affektiven Störungen (20,5\%), insbesondere Depressionen oder Angststörungen (20,5\%). Umgekehrt zeigte sich bspw. in einer studentischen Population, dass Suizidalität bei Patienten mit Major Depression (zu 20\%), Panikstörung (zu 20,6\%) und Generalisierte Angststörung (zu 15,8\%) auftrat (Eisenberg et al. [72]).

Bei Internetabhängigkeit treten wahrscheinlich besonders dann suizidale Gedanken und Impulse auf, wenn es zu belastenden negativen Folgen durch die Sucht kommt, bspw. bei Verlust eines Partners, eines Ausbildungs- oder Arbeitsplatzes oder wenn es im Entzug zu einer depressiven oder auch aggressiven Krise kommt. Die erhöhte Suizidalität bei Internetabhängigen allein auf etwaige psychische Vorerkrankungen zurückzuführen, erscheint jedoch als verkürzt, zumal bekannt ist, dass bei anderen Suchterkrankungen wie z. B. bei Alkoholabhängigkeit [73] und pathologischem Glücksspiel [74] die Suizidgefahr auch unabhängig von der Komorbidität erhöht ist. Dies liegt nicht zuletzt daran, dass sich eine Depressivität gerade dann einstellen kann, wenn eine Suchterkrankung zu gravierenden negativen Auswirkungen im Alltag der Betroffenen geführt hat. Dies gilt gleichsam für die Internetabhängigkeit, die z. T. gravierende leistungsbezogene, soziale und körperliche Konsequenzen nach sich zieht [3]. Bisweilen tritt sie ganz besonders dann in erheblichem Ausmaß in Erscheinung, wenn im Zuge der Abstinenz die akute Sucht überwunden und ihr nachhaltiger Schaden bewusst wird. Der aktuelle wissenschaftliche Stand spricht allerdings dafür, dass Depressivität sowohl primär im Sinne eines Risikofaktors als ein Auslöser fungieren als auch die sekundäre Folge einer IA sein beziehungsweise sich parallel zu ihr entwickeln kann. Unabhängig von kausalen Bezügen sind lebensmüde Gedanken in jedem Fall als ein wichtiger Indikator für eine depressive Entwicklung im Zuge eines problematischen oder pathologischen Internetgebrauchs zu sehen.

\section{Suizidale Handlungen bei Internetabhängigkeit}

In der Zusammenschau stellt sich das Bild für konkrete Suizidalität weniger einheitlich dar. Lediglich in 8 von elf Studien, die auf konkrete suizidale Handlungen bei IA abhoben, zeigten sich signifikante Zusammenhänge, dies jedoch stets in dem Sinne, dass Internetabhängige vergleichsweise häufig von Suizidversuchen berichten. Auch hier spielen komorbide Störungen wie Depressionen, Angsterkrankungen, ADHS und substanzgebundene Suchterkrankungen eine Rolle, wobei lediglich für den Konsum illegaler Drogen ein direkter Effekt auf das Suizidrisiko ermittelt wurde [59]. Suizidale Handlungen können als Fluchtversuch aus einer als nicht mehr lebenswert empfundenen wirklichen Lebensumwelt verstanden werden. Die exzessive Internetnutzung ist als ein scheiternder Versuch der Betroffenen zu sehen, der als kränkend und verunsichernd erlebten konkret-realen Welt zu entfliehen, ohne sich dabei das Leben ganz nehmen zu müssen. Wenn jedoch bei Internetabhängigen im Sinne eines Circulus vitiosus die wirkliche Existenz der Betroffenen durch die Sucht immer weiteren körperlichen und psychosozialen Schaden nimmt, ist zumindest mit einer erhöhten Suizidgefahr zu rechnen. - Die aktuelle Studienlage reicht allerdings nicht aus, um die Frage zu beantworten, ob in der Gruppe der Internetabhängigen tatsächlich von einer generell erhöhten Suizidrate auszugehen ist. 
Unabhängig von den komplexen und bislang noch weitgehend ungeklärten kausalen Zusammenhängen sollte die Ansprache suizidaler und selbstverletzender Impulse und Verhaltensweisen bereits jetzt ein fester Bestandteil in der Diagnostik und Therapie von Internetabhängigkeit werden. In der zeitlich und räumlich verorteten therapeutischen Beziehung können die Betroffenen neue Erfahrungen machen. Auf diesem Weg erschließt Psychotherapie Internetabhängigen echte Spielräume in der unmittelbaren Begegnung mit dem Anderen und der eigenen Leiblichkeit, um der Weltflucht etwas entgegenzusetzen und echte Freude in der Wirklichkeit erlebbar zu machen.

\section{Limitationen}

Ein grundsätzliches Problem dieser Übersichtsarbeit liegt darin begründet, dass anhand der ermittelten Studien lediglich Aussagen über selbstverletzendes Verhalten, suizidale Gedanken und Suizidversuche gemacht werden können, nicht aber über vollendete Suizide. Ähnliches gilt auch für die dokumentierten Todesfälle durch mehrtägigen ununterbrochenen Online-Computerspielkonsum aufgrund von Flüssigkeits- und Schlafmangel [75, 76]. Analog zu den letalen Gefahren substanzgebundener Sucht kann eine „Überdosis“ auch als selbstverletzendes Verhalten mit Todesfolge verstanden werden, wobei hier in der Regel nicht trennscharf zwischen Inkaufnahme oder Absicht unterschieden werden kann.

Da die Online-Nutzungszeit als alleiniges Kriterium für die Diagnosestellung einer IA nachweislich nicht hinreichend ist [10-12], wurden in dieser Übersichtsarbeit nur Studien eingeschlossen, die die IA mit validierten psychometrischen Instrumenten erheben, was die Anzahl eingeschlossener Studien und damit die Aussagekraft eingrenzt. Die Tatsache, dass die IA und ihre Subtypen bislang keinen abschließenden Eingang in die Klassifikationssysteme des ICD 10 [4] beziehungsweise des DSM-5 [5] gefunden haben, schlägt sich auch in der Verwendung einer heterogenen Nomenklatur und einer Vielzahl unterschiedlicher psychometrischer Instrumente nieder. So wird neben dem Begriff der Internetabhängigkeit auch von einem pathologischen oder problematischen Internetgebrauch gesprochen. Neben der Unterteilung in nicht-abhängiges und abhängiges Nutzungsverhalten wird in vielen Studien ein Übergangsbereich beschrieben, der als riskantes [64], maladaptives [65] oder problematisches Verhalten [53] bezeichnet wird und ebenfalls keiner einheitlichen Definition unterliegt.

Ferner ist zu berücksichtigen, dass die Methodik der Studien auch für suizidales Verhalten z. T. unterschiedliche Definitionen zugrunde legen. Dies schränkt die Vergleichbarkeit der Probandengruppen ein. Die Heterogenität der einzelnen Studienergebnisse dürfte sich zudem darauf begründen, dass sowohl die Verbreitung des Internets als auch das Vorkommen von Suizidalität kulturellen Einflüssen der jeweiligen Länder unterliegt. Die vergleichsweise kleine Auswahl an Studien aus sehr unterschiedlichen Regionen kann insofern kein repräsentatives Bild ergeben.

Weitere Limitationen liegen darin begründet, dass die Untersuchungen keine Aussagen darüber zulassen, inwieweit sich Suizidalität und IA gegenseitig kausal bedingen und welche Rolle die komorbiden Erkrankungen spielen. Ob die Depressivität und Suizidalität einen unabhängigen, jedoch verstärkenden Begleitfaktor, eine kausale Ursache oder eine weitere Konsequenz der IA darstellen, wird in longitudinalen Studien an größeren klinischen und nicht- klinischen Kohorten zu überprüfen sein. In diesem Zusammenhang erscheint es sinnvoll, auch die Rolle von Persönlichkeitsfaktoren und -störungen stärker mit in den Blick zu nehmen.

\section{FAZIT FÜR DIE PRAXIS}

Insbesondere im Entzug ist auf suizidale Krisen acht zu geben, wenn die starke Identifikation mit den digitalen Stellvertretern in Avataren und Accounts wegfällt und negative Folgen der Sucht sichtbar werden. In der Psychotherapie ist die Bedeutung suizidaler Gedanken herauszuarbeiten, um zu verstehen, warum der Internetabhängige das Interesse an der konkreten Lebenswirklichkeit und den Menschen im Umfeld verloren hat. Wenn suizidale Impulse verdrängt werden, kann die Verlagerung des Lebensalltags auf eine virtuelle Ebene als neurotischer Konfliktlösungsversuch interpretiert werden. Der spielerische, erotische oder soziale Charakter der jeweiligen Parallelwelten ist dann individuell deutbar.

\section{Interessenkonflikt}

Die Autoren geben an, dass kein Interessenkonflikt besteht.

Literatur

[1] Group MM. Internet Usage and World Population Statistics. http:// www.internetworldstats.com/stats.htm 2016;

[2] Young KS. Internet addiction: The emergence of a new clinical disorder. Cyberpsychol Behav Soc Netw 1998; 1: 237-244

[3] Brand M, Klimmt C, Krämer $\mathrm{N}$ et al. Neue elektronische Medien und Suchtverhalten. In: Ambulanz für Spielsucht (Ambulanz für Spielsucht KuPf, Psychosomatische Medizin und Psychotherapie UdJ, Mainz). Mainz: G-U; 2015

[4] Weltgesundheitsorganisation DH, Mombour W, Schmidt M. Internationale Klassifikation psychischer Störungen ICD-10 Kapitel V (F). Klinisch-diagnostische Leitlinien Bern; Huber: 2005

[5] American Psychiatric Association (Hrsg.) Diagnostic and statistical manual of mental disorders (DSM-5®). Arlington: American Psychiatric Association; 2013

[6] Griffiths M. A 'components' model of addiction within a biopsychosocial framework. J Subst Use 2005; 10: 191-197

[7] Rehbein F, Te Wildt B, Pfeiffer R et al. Positionspapier. Diagnostik von Computerspielabhängigkeit. Fachverband Medienabhängigkeit e. V.; 2010; www.fv-medienabhaengigkeit.de/fileadmin/images/Dateien/ Positionspapier_Diagnostik.pdf

[8] Tao R, Huang X, Wang J et al. Proposed diagnostic criteria for internet addiction. Addiction 2010; 105: 556-564

[9] Griffiths MD. Diagnosis and management of video game addiction. New directions in addiction treatment and prevention 2008; 12 : 27-41

[10] Gentile DA, Choo H, Liau A et al. Pathological video game use among youths: a two-year longitudinal study. Pediatrics 2011; 127 : e319-e329

[11] Van Rooij AJ, Prause N. A critical review of "Internet addiction" criteria with suggestions for the future. J Behal Addict 2014; 3: 203-213 
[12] Gamito PS, Morais DG, Oliveira JG et al. Frequency is not enough: Patterns of use associated with risk of Internet addiction in Portuguese adolescents. Comput Human Behav 2016; 58: 471-478

[13] Przepiorka A, Blachnio A. Time perspective in Internet and Facebook addiction. Comput Human Behav 2016; 60: 13-18

[14] Falkai P, Wittchen H. Diagnostisches und Statistisches Manual Psychiatrischer Störungen DSM-5. Göttingen: Hogrefe; 2015

[15] Bischof G, Bischof A, Meyer C et al. Prävalenz der Internetabhängigkeit-Diagnostik und Risikoprofile (PINTA-DIARI). Lübeck: Kompaktbericht an das Bundesministerium für Gesundheit; 2013

[16] Carli V, Durkee T, Wasserman D et al. The association between pathological internet use and comorbid psychopathology: a systematic review. Psychopathology 2013; 46: 1-13

[17] Pies R. Should DSM-V designate" Internet addiction" a mental disorder? Psychiatry 2009; 6: 31-37

[18] Greenfield DN. Psychological characteristics of compulsive Internet use: A preliminary analysis. Cyberpsychol Behav Soc Netw 1999; 2: 403-412

[19] Shapira NA, Goldsmith TD, Keck PE et al. Psychiatric features of individuals with problematic internet use. J Affect Disord 2000; 57: 267-272

[20] Ho RC, Zhang MW, Tsang TY et al. The association between internet addiction and psychiatric co-morbidity: a meta-analysis. BMC Psychiatry 2014; 14: 183

[21] Ko C-H, Yen J-Y, Yen C-F et al. The association between Internet addiction and psychiatric disorder: a review of the literature. Eur Psychiatry 2012; 27: 1-8

[22] Lee J-Y, Park E-J, Kwon M et al. The difference in comorbidities and behavioral aspects between Internet abuse and Internet dependence in Korean male adolescents. Psychiatry Investig 2014; 11: 387-393

[23] Dieris-Hirche J, Bottel L, Bielefeld $M$ et al Media use and Internet addiction in adult depression: A case-control study. Comput Human Behav 2017; 68: 96-103

[24] O'Carroll PW, Berman AL, Maris RW et al. Beyond the Tower of Babel: a nomenclature for suicidology. Suicide Life Threat Behav 1996; 26: 237-252

[25] Silverman MM, Berman AL, Sanddal ND et al. Rebuilding the tower of Babel: a revised nomenclature for the study of suicide and suicidal behaviors. Part 2: Suicide-related ideations, communications, and behaviors. Suicide Life Threat Behav 2007; 37: 264-277

[26] Silverman MM, Berman AL, Sanddal ND et al. Rebuilding the tower of babel: a revised nomenclature for the study of suicide and suicidal behaviors part 1 : background, rationale, and methodology. Suicide Life Threat Behav 2007; 37: 248-263

[27] Arsenault-Lapierre G, Kim C, Turecki G. Psychiatric diagnoses in 3275 suicides: a meta-analysis. BMC Psychiatry 2004; 4: 37

[28] Mann J]. Neurobiology of suicidal behaviour. Nat Rev Neurosci 2003; 4: 819-828

[29] Joiner TE, Buchman-Schmitt JM, Chu C. Do Undiagnosed Suicide Decedents Have Symptoms of a Mental Disorder? J Clin Psychol 2017, epub doi:10.1002/jclp.22498

[30] Yuodelis-Flores C, Ries RK. Addiction and suicide: A review. Am Addict 2015; 24: 98-104

[31] Newman SC, Thompson AH. A population-based study of the association between pathological gambling and attempted suicide. Suicide Life Threat Behav 2003; 33: 80-87

[32] Petermann F, Nitkowski D. Selbstverletzendes Verhalten. Nervenarzt 2008; 79: 1017-1022

[33] Dougherty DM, Mathias CW, Marsh DM et al. Laboratory measured behavioral impulsivity relates to suicide attempt history. Suicide Life Threat Behav 2004; 34: 374-385
[34] Kamphuis JH, Ruyling SB, Reijntjes AH. Testing the emotion regulation hypothesis among self-injuring females: Evidence for differences across mood states. J Nerv Ment Dis 2007; 195: 912-918

[35] Lloyd-Richardson EE, Perrine N, Dierker L et al. Characteristics and functions of non-suicidal self-injury in a community sample of adolescents. Psychol Med 2007; 37: 1183-1192

[36] O'Connor RC, Nock MK. Suicide 2 The psychology of suicidal behaviour. Lancet Psychiatry 2014; 1: 73-85

[37] Joiner T. Why people die by suicide. Cambridge, Mass: Harvard University Press; 2007

[38] Kessler RC, Ustun TB. The WHO world mental health surveys: Global Perspectives on the Epidemiology of Mental Disorders. New York: Cambridge University Press; 2008

[39] Bertolote JM, Fleischmann A, Butchart A et al. Suicide, suicide attempts and pesticides: a major hidden public health problem. Bull World Health Organ 2006; 84: 260-260

[40] World Health Organization. Preventing suicide: A global imperative. World Health Organization; 2014

[41] Patton GC, Coffey C, Sawyer SM et al. Global patterns of mortality in young people: a systematic analysis of population health data. Lancet 2009; 374: 881-892

[42] Eichenberg C. Empirische Befunde zu Suizidforen im Internet. Suizidprophylaxe 2010; 37: 67-74

[43] Brand M, Young KS, Laier C et al. Integrating psychological and neurobiological considerations regarding the development and maintenance of specific Internet-use disorders: An Interaction of Person-Affect-Cognition-Execution (I-PACE) model. Neurosci Biobehav Rev 2016; 71: 252-266

[44] Davis RA. A cognitive-behavioral model of pathological Internet use. Comput Human Behav 2001; 17: 187-195

[45] Cao F, Su L, Liu T et al. The relationship between impulsivity and Internet addiction in a sample of Chinese adolescents. Eur Psychiatry 2007; 22: 466-471

[46] Hawton K, Rodham K, Evans E et al. Deliberate self harm in adolescents: self report survey in schools in England. BMJ 2002; 325: $1207-1211$

[47] Kuss DJ, Griffiths MD. Internet gaming addiction: A systematic review of empirical research. Int J Ment Health Addict 2012; 10: 278-296

[48] Franklin JC, Ribeiro JD, Fox KR et al. Risk factors for suicidal thoughts and behaviors: A meta-analysis of 50 years of research. Psychol Bull 2017; 143: 187-232

[49] Durkee T, Hadlaczky G, Westerlund M et al. Internet pathways in suicidality: a review of the evidence. Int J Environ Res Public Health 2011; 8: 3938-3952

[50] Daine K, Hawton K, Singaravelu V et al. The power of the web: a systematic review of studies of the influence of the internet on self-harm and suicide in young people. Plos One 2013; 8: e77555e77555

[51] Lam LT, Peng Z, Mai ] et al. The association between internet addiction and self-injurious behaviour among adolescents. Inj Prev 2009; 15: 403-408

[52] Messias E, Castro J, Saini A et al. Sadness, suicide, and their association with video game and internet overuse among teens: results from the youth risk behavior survey 2007 and 2009. Suicide Life Threat Behav 2011; 41: 307-315

[53] Fu K-w, Chan WSC, Wong PWC et al. Internet addiction: prevalence, discriminant validity and correlates among adolescents in Hong Kong. Br J Psychiatry 2010; 196: 486-492

[54] Kim K, Ryu E, Chon M-Y et al. Internet addiction in Korean adolescents and its relation to depression and suicidal ideation: a questionnaire survey. Int J Nurs Stud 2006; 43: 185-192 
[55] Alpaslan AH, Soylu N, Avci K et al. The association between problematic internet use, suicide probability, alexithymia and loneliness among Turkish medical students. Klin Psikofarmakol B 2013; 23: S115-S115

[56] Alpaslan AH, Soylu N, Kocak U et al. Problematic Internet use was more common in Turkish adolescents with major depressive disorders than controls. Acta Paediatr 2016; 105: 695-700

[57] Kurt DG. Suicide risk in college students: The effects of Internet addiction and drug use. Kuram Uygul Egit Bil 2015; 15: 841-848

[58] Oktan V. An investigation of problematic internet use among adolescents in terms of self-injurious and risk-taking behavior. Child Youth Serv Rev 2015; 52: 63-67

[59] Park S, Hong K-EM, Park EJ et al. The association between problematic internet use and depression, suicidal ideation and bipolar disorder symptoms in Korean adolescents. Aust N Z J Psychiatry 2013; 47: 153-159

[60] Wang P-W, Liu T-L, Ko C-H et al. Association between Problematic Cellular Phone Use and Suicide: The Moderating Effect of Family Function and Depression. Compr Psychiatry 2014; 55: 342-348

[61] Wu C-Y, Lee M-B, Liao S-C et al. Risk Factors of Internet Addiction among Internet Users: An Online Questionnaire Survey. Plos One 2015; 10: e0137506-e0137506

[62] Kim B-S, Chang SM, Park JE et al. Prevalence, correlates, psychiatric comorbidities, and suicidality in a community population with problematic Internet use. Psychiatry Res 2016; 244: 249-256

[63] Lin IH, Ko C-H, Chang Y-P et al. The association between suicidality and Internet addiction and activities in Taiwanese adolescents. Compr Psychiatry 2014; 55: 504-510

[64] Fischer G, Brunner R, Parzer P et al. Depressivität, selbstverletzendes und suizidales Verhalten bei Jugendlichen mit riskanter und pathologischer internetnutzung. $=$ Depression, deliberate self-harm and suicidal behaviour in adolescents engaging in risky and pathological internet use. Prax Kinderpsychol Kinderpsychiatr 2012; 61: 16-31

[65] Kaess M, Durkee T, Brunner R et al. Pathological Internet use among European adolescents: psychopathology and self-destructive behaviours. Eur Child Adolesc Psychiatry 2014; 23: 1093-1102

[66] Rikkers W, Lawrence D, Hafekost J et al. Internet use and electronic gaming by children and adolescents with emotional and behavioural problems in Australia - results from the second Child and Adolescent Survey of Mental Health and Wellbeing. BMC Public Health 2016; 16 : 399-399
[67] Strittmatter E, Kaess M, Parzer P et al. Pathological Internet use among adolescents: Comparing gamers and non-gamers. Psychiatry Res 2015; 228: 128-135

[68] Dalbudak E, Evren C, Aldemir S et al. The severity of Internet addiction risk and its relationship with the severity of borderline personality features, childhood traumas, dissociative experiences, depression and anxiety symptoms among Turkish university students. Psychiatry Res 2014; 219: 577-582

[69] Wu JY-W, Ko H-C, Tung Y-Y et al. Internet use expectancy for tension reduction and disinhibition mediates the relationship between borderline personality disorder features and Internet addiction among college students-One-year follow-up. Comput Human Behav 2016 55: 851-855

[70] Müller A, Claes L, Smits D et al. Prevalence and correlates of self-harm in the German general population. PLoS one 2016; 11: e0157928

[71] Nock MK, Hwang I, Sampson N et al. Cross-national analysis of the associations among mental disorders and suicidal behavior: findings from the WHO World Mental Health Surveys. Plos Med 2009; 6: e1000123

[72] Eisenberg D, Gollust SE, Golberstein E et al. Prevalence and correlates of depression, anxiety, and suicidality among university students. Am J Orthopsychiatry 2007; 77: 534-542

[73] Darvishi N, Farhadi M, Haghtalab T et al. Alcohol-related risk of suicidal ideation, suicide attempt, and completed suicide: a meta-analysis. PloS one 2015; 10: e0126870

[74] Battersby M, Tolchard B, Scurrah M et al. Suicide ideation and behaviour in people with pathological gambling attending a treatment service. Int J Ment Health Addict 2006; 4: 233-246

[75] Christakis DA. Internet addiction: a 21 st century epidemic? BMC Medicine 2010; 8: 61

[76] Golub A, Lingley K. 'Just like the Qing Empire:' Internet addiction, MMOGs, and moral crisis in contemporary China. Games and Cult 2008; 3: 59-75 